\title{
Shear Wave Elastography (SWE) Evaluation of Asymptomatic Flexor Pollicis Longus Tendon Condition After Volar Plate Fixation for Distal Radius Fracture
}

Xiaodong Bai (D)

Zhenyu Liu*

Baojun Wang*

Department of Orthopedics, Beijing Friendship Hospital, Capital Medical University, Beijing 100050, People's Republic of China

*These authors contributed equally to this work
This article was published in the following Dove Press journal:

International Journal of General Medicine

\author{
International journal of General Medicine
}

\begin{abstract}
Objective: This study aimed to evaluate asymptomatic flexor pollicis longus (FPL) tendon condition after volar plate fixation for distal radius fracture using shear wave elastography (SWE). Methods: We evaluated 36 distal radius fractures with shear wave elastography to evaluate the FPL tendon after volar plate fixation. Ultrasonography was used to measure the FPL distance to the volar plate in the involved wrists, and the thickness of the FPL tendon in the involved wrists was measured on SWE sonograms taken 12 months postoperatively. Measurements of the involved wrists were compared with those of the intact wrists. The radiographic parameters and Soong grade were collected to analyze the correlation between these indicators and the value of the Young's modulus and asymptomatic tendon injury.

Results: The mean values of the Young's modulus for involved wrist were lower than for intact wrist tendons. The values of the Young's modulus were correlated with the FPL distance, radial inclination, and Soong grade. FPL tendons of involved wrists exhibited in the majority a yellow (intermediate) SWE signal, compared to intact wrist tendons, which exhibited a red (hard) signal in the vast majority, and there were significant differences between the involved and intact wrist.
\end{abstract}

Conclusion: FPL tendon may develop asymptomatically changes after volar locking plate fixation of distal radius fractures, which can be detected by SWE effectively. FPL distance, radial accretion, and Soong grade may be the main causes of asymptomatic FPL tendon erosion.

Keywords: distal radius fractures, shear wave elastography, flexor pollicis longus tendon, Soong grade, asymptomatic tendon injury

\section{Introduction}

Distal radius fractures are a common fracture of the upper extremity. Recently, volar locking plates have been the most common treatment for open reduction and internal fixation of distal radius fractures. Flexor tendon rupture or irritation is the most common complication, especially the flexor pollicis longus (FPL) damage. ${ }^{1-4}$ However, there is no effective method for early evaluation of tendon injury.

Shear wave elastography (SWE) is a real-time diagnostic imaging technique, it can use ultrasound to quantitatively assess differences in tissue stiffness. In previous studies, SWE has been used to evaluate the stiffness of some organs, such as liver, breast, prostate, and thyroid lesions. ${ }^{5-7}$ Recently, some studies showed that SWE can be used in musculoskeletal evaluation, especially for characterization and
Correspondence: Zhenyu Liu; Baojun Wang Email doczhyl@।63.com;

docbjwang@I26.com
International Journal of General Medicine 2021:14 187-193 
differentiation between normal and damaged tendons. ${ }^{8-10}$ SWE appears to be a utility tool to improve the diagnosis of tendon injury, and it can also be used as an evaluation index for therapeutic effects. But there is no previous study evaluating FPL after ORIF.

Asymptomatic tendon injury means that the tendon does not break or rupture, and the patient does not have local pain weakness and other discomfort, but the patient's tendon has shown elastic changes and edema performance. Traditional ultrasound can reflect the degree of asymptomatic tendon injury to a certain extent, but it is unable to quantify the degree of injury. To our knowledge, there have been no reports on using SWE to assess asymptomatic flexor tendon injury after internal fixation of distal radius fractures. This study was to use SWE to evaluate FPL damage and demonstrate the feasibility and the value of the Young's modulus in the application of asymptomatic tendon damage after volar locking plate fixation of distal radius fractures.

\section{Materials and Methods}

This retrospective study was conducted at a single university teaching center. Thirty-six patients with distal radius fractures who underwent open reduction and internal fixation with the radius distal volar locking plate were retrospectively recruited from 2016-2019. Included patients had a mean age of 62.8 years (range $=52-73$ years) at the time of surgery. There were 11 males and 25 females. Twenty wrists were on the right side and 16 wrists were on the left side. Patients were asked to return for a followup visit after surgery. In our hospital, we have established a register system for all the patients with surgery. In addition, special personnel are responsible for regular appointments of patients for follow-up. Informed consent was obtained from each patient before enrollment, and the study was approved by our institutional review board.

All the patients in this study were approximately a year after operation and the fracture healed completely. The patients without symptomatic flexor tenosynovitis (patients had swelling on the volar side of the wrist and pain during thumb movements) were included for our study. Exclusion criteria for all participants were the following: Rheumatoid arthritis, open fracture, fracture of distal ulna, infections, bone nonunion, diabetes, and collagen tissue disease.

We reviewed the records of all included patients with Arbeitsgemeinschaftfür Osteosynthesefragen (AO) classification. All involved patients underwent open reduction and internal fixation with the radius distal volar locking plate ( $2.4 \mathrm{~mm}$, variable angle LCP volar rim distal radius plate, all plates used are from the same manufacturer) in our hospital. All operations were performed by the same group of doctors. In this study, the standard volar approach of the wrist joint was taken in all surgeries, during the operation: after exposing the flexor carpi radialis, open its tendon sheath, expose the flexor pollicis longus, and pull it to the radial side after opening the deep fascia. At the same time, pay attention to protect the median nerve and radial artery. At this time, the pronator quadratus muscle can be exposed. In some cases with obvious fracture displacement, we can find that the integrity of the pronator quadratus muscle is destroyed by the displaced metacarpal fracture block. When the pronator quadratus muscle was fully exposed, the pronator quadratus muscle was cut transversely only at the height of the "watershed" at the distal radius. At this level, the $0.5 \mathrm{~cm}$ pronator quadratus muscle was cut longitudinally along the radial insertion point of the pronator quadratus muscle, The space between the pronator quadratus and the radius was opened with a wide periosteum stripper. Attention should be paid to the protection of the pronator quadratus muscle when the fracture ends were reduced and the volar plate was fixed When the screw is used, a $0.2 \mathrm{~mm}$ incision can be made on the surface of the pronator quadratus muscle parallel to the muscle fiber, and the drilling screw placement can be completed by using a soft tissue protector. After the wound is washed, the muscle tissue of pronator quadratus transversely cut at the height of "watershed" is sutured, and the surgical incision is sutured without the placement of a drainage strip, and there is no auxiliary plaster external fixation after the operation.

The following radiographic parameters were collected in imaging examination at 12 months after operation, including: palmar tilt, radial inclination, radial height, and Soong grade. All the X-ray films were standardized to accommodate any difference in radiographic magnification. Five independent observers (all senior orthopedics trainees) blind to case-control status reviewed each of the radiographs. Every observer was instructed to measure some of the parameters in radiographs. On the AP radiograph, the radial inclination and radial height were measured. On the lateral view, the palmar tilt and Soong grade were noted and measured. Radiological results were evaluated with Lidstrom scores modified by Sarmiento et al. ${ }^{11}$ We used Soong's classification to evaluate the volar plate prominence. ${ }^{4}$ Soong's classification grade 0 : the position of the distal radius plates did not project past the radius volar rim line, grade 1: plates that projected volar rim line but proximal to the volar rim, and grade 2: plates that projected directly on or beyond the volar rim line. 


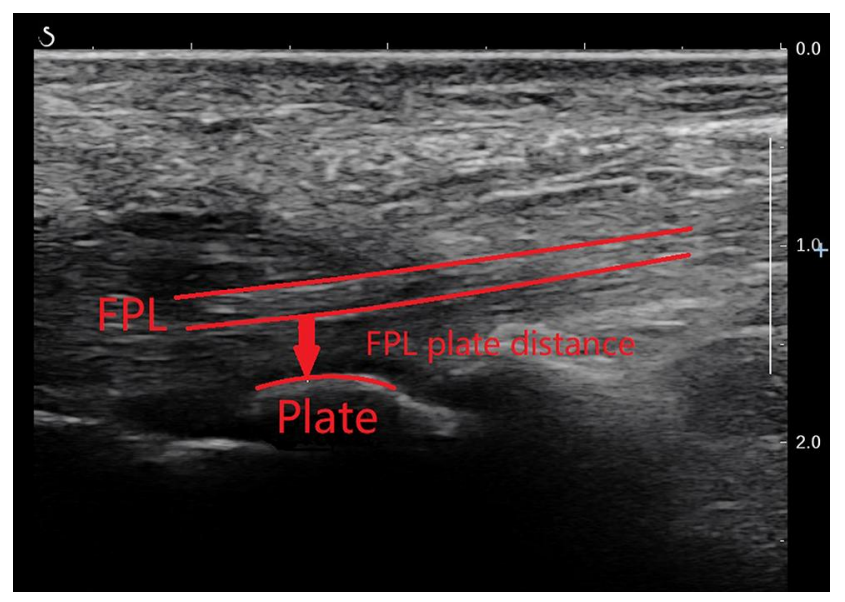

Figure I FPL plate distance. It is defined as the distance between the distal edge of the plate and the FPL tendon.

We used conventional ultrasound and SWE to evaluate the FPL in the course of the patient's recovery. Two radiologists with at least 5 years of experience in sonographic imaging and SWE application performed all the examinations. Every patient underwent the same entire US protocol for both left- and right-sided tendons. The patients were randomized according to the side with which the examination was started. Flexor pollicis longus (FPL) plate distance was assessed by conventional ultrasound (Figure 1). A standardized positioning protocol was applied to each patient, as shown below. All US studies were carried out in the same order on a dedicated sonography unit. (My Lab Five; Esaote, Florence, Italy). The wrist was put in the neutral position, and the ultrasound probe was placed on the volar side at the level of the radiocarpal joint on the transverse plane. The identity of the FPL tendon was confirmed with the help of active thumb extension. The probe was rotated into the longitudinal axis. The minimum distance between the plate and the FPL tendon was measured. Patient reports and clinical findings were recorded.

Semiquantitative evaluation of tendon stiffness was by SWE. ${ }^{12}$ FPL tissue rigidity was assessed semiquantitatively by color charts. In FPL, three equal Q-box measurements $\left(1 \mathrm{~cm}^{2}\right)$ were performed in the proximal, mid-, and distal tendon parts of the plate (sagittal view). The SWE measurements were performed in the distal area of the plate. FPL tissue rigidity was assessed by SWE. Tendon stiffness then was grouped into "soft" (Figure 2), "intermediate" (Figure 3), or "hard" (Figure 4), according to the color of region of interest (ROI).

Continuous variables are presented as mean \pm standard deviation (SD). Categorical variables are presented as frequency and relative frequencies. The paired Student $t$-test was used to evaluate differences of quantitative values of the Young's modulus between the involved and intact wrist for data with normal distribution or Wilcoxon signed-rank test for data with not normal distribution. Pearson's correlation analysis and multiple stepwise simple linear regression analysis were performed to correlate the value of the Young's modulus difference with the respective variables, including the distance between the FPL tendon and distal edge of the plates, Soong grade, palmar tilt, radial inclination, and radial height. All statistical analyses were performed

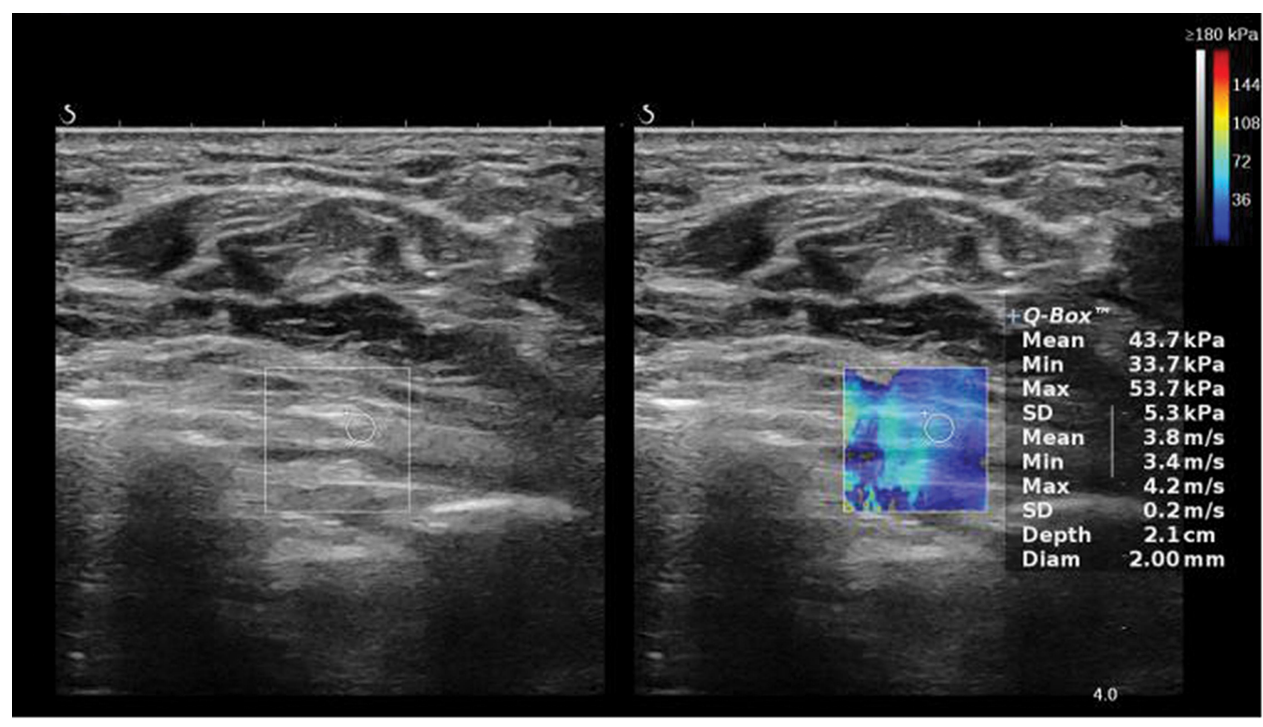

Figure 2 FPL tissue rigidity was assessed by SWE, the mean Young's modulus was $43.7 \mathrm{kPa}$, and the color of ROI was blue, which means the rigidity of FPL was soft. 


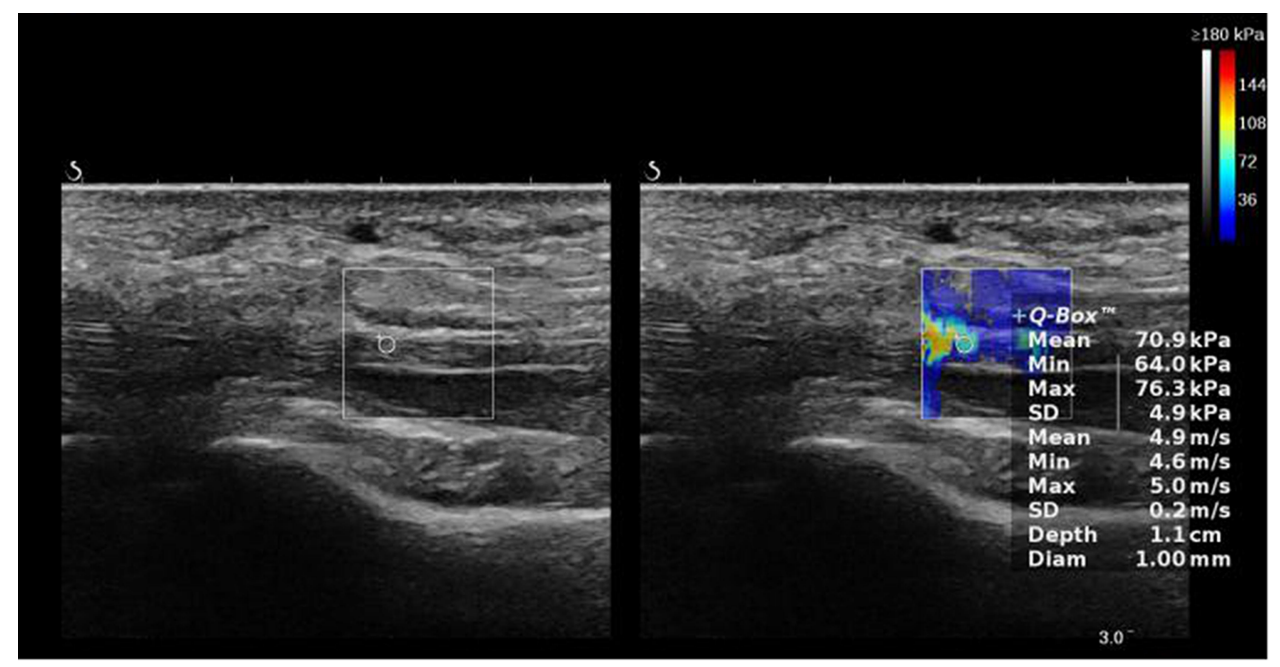

Figure 3 FPL tissue rigidity was assessed by SWE, the mean Young's modulus was $70.9 \mathrm{kPa}$, and the color of ROI was yellow, which means the rigidity of FPL was intermediate.

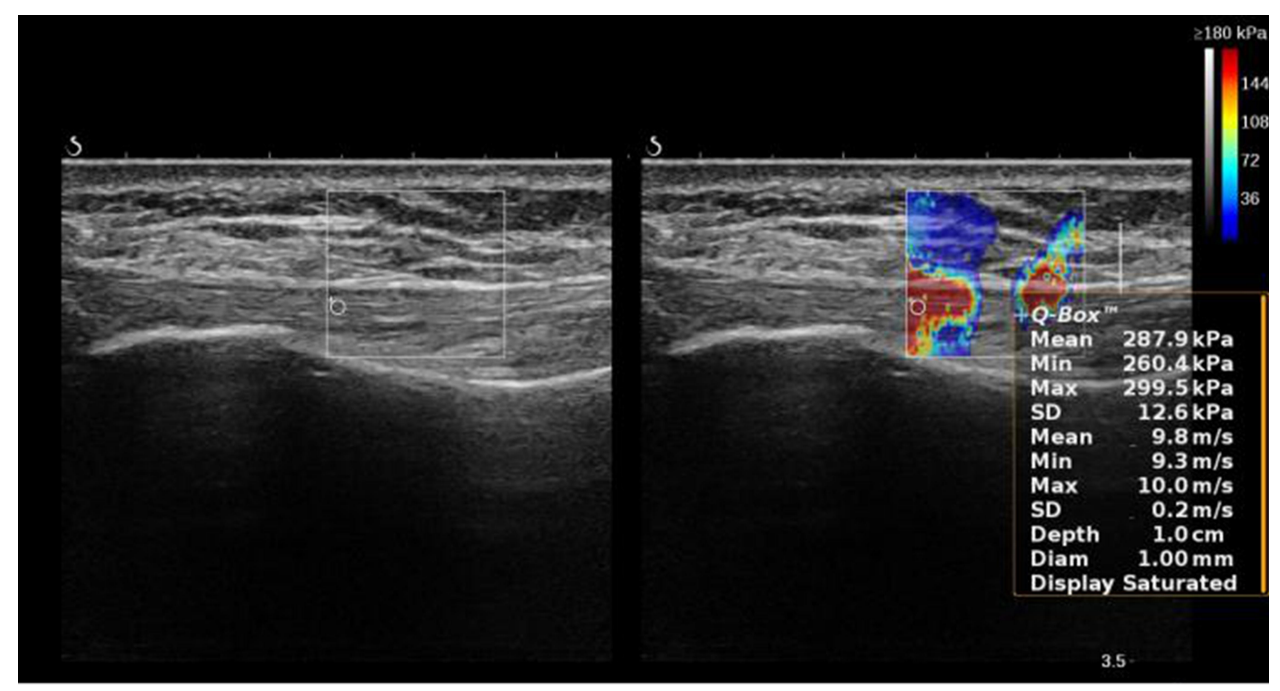

Figure $4 \mathrm{FPL}$ tissue rigidity was assessed by SWE, the mean Young's modulus was $287.9 \mathrm{kPa}$, and the color of ROI was red, which means the rigidity of FPL was hard.

using the SPSS Statistics version 22 (IBM Corp., Armonk, NY). Statistical significance was defined as $P<0.05$.

\section{Results}

Overall, a total of 36 wrists from 36 patients with distal radius fractures who received volar locking plates without residual symptoms agreed to this study. Of these 36 fractures, there were seven 31-A1, 14 31-A2, 70 31-A3, and eight 31-A3 fractures according to AO classification. Of Soong grade, 12 plates were classified as Soong grade 0, 17 as Soong grade 1, and seven as Soong grade 2. Mean time between surgery and follow-up was 15.2 months (range $=12-23$ months).
For quantitative analysis of the values of the Young's modulus, the mean value of the Young's modulus for the involved wrist was significantly lower than for intact wrist tendons with normal distribution $(P=0.001)$. The mean value of the Young's modulus was $255.4 \mathrm{kPa}$ (range=179.6-302.5 kPa) for the involved wrist and $285.1 \mathrm{kPa}$ (range $=264.7-307.7 \mathrm{kPa}$ ) for the intact wrist tendons. In the involved wrist, there were no significant differences in the value of Young's modulus with regard to Soong grade (Soong grade 0: $270.8 \pm 21.1 \mathrm{kPa}$, grade 1: $251.7 \pm 30.2 \mathrm{kPa}$, and grade 2: $237.9 \pm 37.6 \mathrm{kPa}$ ). The results of Pearson's correlation analysis indicate that the values of the Young's modulus were significantly correlated with the FPL distance $(P=0.001, \mathrm{r}=0.914)$, radial inclination $(P=0.001$, 
Table I Correlation Between SWE Involved Wrist and Parameters

\begin{tabular}{|l|l|l|l|}
\hline Parameter & Value & Spearman $(\boldsymbol{r}, \boldsymbol{P})$ & Multiple Analysis $(\boldsymbol{P})$ \\
\hline Age & $61.6(47-73)$ & $-0.025,0.883$ & 0.904 \\
Palmar tilt & $8.5(13.0-0.0)$ & $0.240,0.159$ & 0.131 \\
Radial inclination & $20.9(18.5-24.3)$ & $0.823,<0.001$ & 0.029 \\
R adial height & $10.9(8.9-13.2)$ & $0.081,0.638$ & 0.271 \\
FPL distance & $0.8(0.2-1.5)$ & $0.914,<0.001$ & $<0.001$ \\
Soong grade & & $-0.394,0.017$ & 0.897 \\
\hline
\end{tabular}

$\mathrm{r}=0.823)$, and Soong grade $(P=0.017, \mathrm{r}=0.39)$. The multiple linear regression analysis confirmed that the value of the Young's modulus was significantly associated with changes in the FPL distance (coefficient $=0.697,95 \%$ confidence interval $=36.644-87.919 ; P=0.001$ ), and radial inclination (coefficient $=0.305, \quad 95 \% \quad$ confidence interval $=0.643-10.811 ; P=0.029)$ (Table 1).

For semi-quantitative analysis of the values of the Young's modulus, FPL tendons of the involved wrist exhibited in the majority a yellow (intermediate) SWE signal $(61.1 \%)$, compared to intact wrist tendons, which exhibited a red (hard) signal in the vast majority $(83.3 \%)$. There were significant differences between the involved and intact wrist.

\section{Discussion}

By the interindividual comparative study, we investigated the feasibility with which SWE helps in depicting asymptomatic tendon injury. This current study demonstrated that SWE may be capable of detecting the change of tendon and providing an aid for further monitoring of the dynamic changes of the asymptomatic tendon injury. Although the involved FPL tendon did not appear with edema, pain, tendinitis, or other clinical symptoms after open reduction and internal fixation, our results showed that the elastic value had changed with the quantitative analysis of the Young's modulus, which was decreased compared to the intact wrists.

Through the analysis of measurement data, we found that there was a correlation between the change of the value of the Young's modulus of FPL and PFL distance. The larger FPL distance in the involved wrist, the closer the value of the Young's modulus to the intact wrist. On the contrary, the smaller the PFL distance, the lower the value of the Young's modulus. Previous literature reports also mentioned this view. ${ }^{13}$ From the anatomical point of view, the most effective space between FPL and the plate is the pronator quadratus muscle. Therefore, the results suggest that we need to preserve and protect the pronator quadratus muscle as much as possible, and repair the damaged pronator muscle effectively during operation.

The FPL value of the Young's modulus is related to Soong grade in the involved wrist. In the study, we also conducted Soong grade analysis after internal fixation. Through statistical analysis, we found that different Soong grade of distal radial plate placement will affect the value of the Young's modulus of FPL in the involved wrist, among which grade A is better than grade B and grade $\mathrm{C}$, which suggests that we should try our best to fix the distal radial plate in the correct position to avoid asymptomatic injury of FPL due to improper plate placement injury.

For the semi-quantitative analysis of the value of the Young's modulus: We classified SWE imaging according to color chart, blue: soft tissue rigidity, yellow: intermediate tissue rigidity, red: hard tissue rigidity. The majority of FPL tendons in the involved wrist showed a yellow (intermediate) SWE signal (61.1\%), while the majority of intact wrist tendons showed red (hard) signals (83.3\%). There was a significant difference between wrist joint involvement and integrity.

SWE is a kind of real-time diagnostic imaging technique, and it uses US to quantitatively assess different tissue stiffness. Real-time ultra-fast SWE measures shear wave velocity generated by the US pulse. SWE has been used to evaluate stiffness of the liver, breast, ${ }^{14}$ prostate, ${ }^{7}$ and thyroid lesions, ${ }^{15}$ and SWE increases the specificity and positive predictive value in these diseases. Recently, studies showed that SWE might be a useful tool for characterization and differentiation tendons. ${ }^{16}$ SWE can evaluate the tendon elasticity to determine whether the tendon stiffness is reduced or not, so as to detect tendon lesions. ${ }^{17,18}$ Studies have shown that SWE changed in the presence of tendon softening, pain, and functional defects. ${ }^{19}$ Our study found that SWE also has a good detection significance in the presence of asymptomatic tendon injury, which can help us better guide the rehabilitation of patients and make decisions on whether to remove the internal 
fixation in time. Therefore, SWE can be used as an effective method for diagnosis of tendinopathies of FPL, especially in asymptomatic cases.

FPL plate distance is defined as the distance between the FPL tendon and distal edge of the plates before plate removal. Tokunaga and $\mathrm{Abe}^{20}$ found that the FPL plate distance was $0 \mathrm{~mm}$ in the tendon damage wrists, which indicated that the FPL tendon was in contact with the volar plate. According to a recent study from Kara, the mean distance between the volar plate and the FPL tendon was $0.3 \mathrm{~mm}$ in the involved wrist, whereas the distance between the FPL tendon and the volar rim in the intact wrist was $1.3 \mathrm{~mm}$. Our measurement result of FPL plate distance is $0.8 \mathrm{~mm}$, and this is related to the fact that we did not completely cut the pronator quadratus muscle during the operation and repaired the pronator quadratus muscle well after the internal fixation.

Soong et $\mathrm{al}^{4}$ graded the plate according to the degree of protrusion in 2011, and studied the correlation with postoperative complications. The results showed that three cases of tendon rupture occurred in 168 cases of distal radius fractures, two cases were classified as Soong grade 2, and one case was Soong grade 1. The results of our study showed that: $47 \%(17 / 36)$ of cases were Soong grade 2, and 19\% (7/36) of cases were Soong grade 1. From the measurement of the value of the Young's modulus, the cases with Soong grade 2 and 3 had no tendon rupture case, but their values of the Young's modulus were more likely to become soft or intermediate tissue rigidity. In general, for the evaluation of the curative effect of surgery for distal radius fractures, we pay attention to the recovery of fracture alignment mainly including: palmar tilt, radial inclination, radial height, and other imaging parameters. Our results suggest that the most significant predictor of asymptomatic FPL injury is radial inclination. Therefore, in the process of internal fixation of distal radius fractures, we should pay attention to good reduction of fractures and correct placement of plates.

Our study has some limitations. The limitations of this study include the fracture pattern of each patient, and we only used SWE to evaluate the FPL tendon, despite the possibility of other tendons being at risk of asymptomatic tendon injury. The sample size was small, although it was enough to find out the statistically significant and clinically important results. In our study, we did not remove the plate after fracture healing to determine whether there were several lesions or injuries under direct vision. However, for the prognosis of flexor tendon, longer follow-up results are still uncertain. In such cases, more follow-up and longterm monitoring may be required.

\section{Abbreviations}

SWE, shear wave elastography; FPL, flexor pollicis longus.

\section{Data Sharing Statement}

Please contact the author for data requests. The data is available from Xiaodong Bai, E-mail: baixiaodong@ccmu.edu.cn.

\section{Ethics Approval and Consent to Participate}

The study was approved by the ethics committee of Beijing Friendship Hospital. This study was conducted in accordance with the latest version of the Declaration of Helsinki. Each subject was ensured anonymity, which was maintained by using subject-specific numeric codes on all records, including registration cards.

\section{Author Contributions}

All authors made substantial contributions to the conception and design, acquisition of data, or analysis and interpretation of data; took part in drafting the article or revising it critically for important intellectual content; agreed to submit to the current journal; gave final approval of the version to be published; and agree to be accountable for all aspects of the work.

\section{Funding}

No funding was received.

\section{Disclosure}

The authors declare that they have no conflicts of interest for this work.

\section{References}

1. Drobetz H, Kutscha-Lissberg E. Osteosynthesis of distal radial fractures with a volar locking screw plate system. Int Orthop. 2003;27 (1):1-6. doi:10.1007/s00264-002-0393-x

2. Arora R, Lutz M, Hennerbichler A, et al. Complications following internal fixation of unstable distal radius fracture with a palmar locking-plate. J Orthop Trauma. 2007;21(5):316-322. doi:10.1097/ BOT.0b013e318059b993

3. Casaletto JA, Machin D, Leung R, et al. Flexor pollicis longus tendon ruptures after palmar plate fixation of fractures of the distal radius. $J$ Hand Surg Eur Vol. 2009;34(4):471-474. doi:10.1177/ 1753193408100964

4. Soong M, van Leerdam R, Guitton TG, et al. Fracture of the distal radius: risk factors for complications after locked volar plate fixation. J Hand Surg Am. 2011;36(1):3-9. doi:10.1016/j.jhsa.2010.09.033 
5. Huang Z, Zheng W, Zhang Y-J, et al. Assessing hepatic fibrosis using 2-D shear wave elastography in patients with liver tumors: a prospective single-center study. Ultrasound Med Biol. 2017;43 (11):2522-2529. doi:10.1016/j.ultrasmedbio.2017.07.003

6. Woo S, Suh CH, Kim SY, et al. Shear-wave elastography for detection of prostate cancer: a systematic review and diagnostic meta-analysis. AJR Am J Roentgenol. 2017;209(4):806-814. doi:10.2214/AJR.17.18056

7. Correas J-M, Tissier A-M, Khairoune A, et al. Prostate cancer: diagnostic performance of real-time shear-wave elastography. Radiology. 2015;275(1):280-289. doi:10.1148/radiol.14140567

8. Leonardis JM, Desmet DM, Lipps DB. Quantifying differences in the material properties of the fiber regions of the pectoralis major using ultrasound shear wave elastography. J Biomech. 2017;63:41-46. doi:10.1016/j.jbiomech.2017.07.031

9. Hatta T, Giambini H, Itoigawa Y, et al. Quantifying extensibility of rotator cuff muscle with tendon rupture using shear wave elastography: a cadaveric study. J Biomech. 2017;61:131-136. doi:10.1016/j. jbiomech.2017.07.009

10. Ono T, Katsura D, Yamada K, et al. Use of ultrasound shear-wave elastography to evaluate change in cervical stiffness during pregnancy. J Obstet Gynaecol Res. 2017;43(9):1405-1410. doi:10.1111/jog.13379

11. Sarmiento A, Pratt GW, Berry NC, et al. Colles' fractures. Functional bracing in supination. The Journal of Bone \& Joint Surgery. 1975;57 (3):311-317. doi:10.2106/00004623-197557030-00004

12. Dirrichs T, Schrading S, Gatz M, et al. Shear wave elastography (SWE) of asymptomatic achilles tendons: a comparison between semiprofessional athletes and the nonathletic general population. Acad Radiol. 2019;26(10):1345-1351. doi:10.1016/j.acra.2018.12.014
13. Kara A, Celik H, Bankaoglu M, et al. Ultrasonic evaluation of the flexor pollicis longus tendon following volar plate fixation for distal radius fractures. J Hand Surg Am. 2016;41(3):374-380. doi:10.1016/ j.jhsa.2015.11.022

14. Athanasiou A, Tardivon A, Tanter M, et al. Breast lesions: quantitative elastography with supersonic shear imaging-preliminary results. Radiology. 2010;256(1):297-303. doi:10.1148/radiol.10090385

15. Ragazzoni F, Deandrea M, Mormile A, et al. High diagnostic accuracy and interobserver reliability of real-time elastography in the evaluation of thyroid nodules. Ultrasound Med Biol. 2012;38 (7):1154-1162. doi:10.1016/j.ultrasmedbio.2012.02.025

16. De Zordo T, Lill SR, Fink C, et al. Real-time sonoelastography of lateral epicondylitis: comparison of findings between patients and healthy volunteers. AJR Am J Roentgenol. 2009;193(1):180-185. doi:10.2214/AJR.08.2020

17. Kot BC, Zhang ZJ, Lee AWC, et al. Elastic modulus of muscle and tendon with shear wave ultrasound elastography: variations with different technical settings. PLoS One. 2012;7(8):e44348. doi:10.1371/journal.pone.0044348

18. Chen X-M, Cui L-G, He P, et al. Shear wave elastographic characterization of normal and torn achilles tendons: a pilot study. J Ultrasound Med. 2013;32(3):449-455. doi:10.7863/jum.2013.32.3.449

19. Ooi CC, Richards PJ, Maffulli N, et al. A soft patellar tendon on ultrasound elastography is associated with pain and functional deficit in volleyball players. J Sci Med Sport. 2016;19(5):373-378. doi:10.1016/j.jsams.2015.06.003

20. Tokunaga S, Abe Y. Asymptomatic flexor tendon damages after volar locking plate fixation of distal radius fractures. J Hand Surg Asian Pac Vol. 2017;22(1):75-82. doi:10.1142/S0218810417500137
International Journal of General Medicine

\section{Publish your work in this journal}

The International Journal of General Medicine is an international, peer-reviewed open-access journal that focuses on general and internal medicine, pathogenesis, epidemiology, diagnosis, monitoring and treatment protocols. The journal is characterized by the rapid reporting of reviews, original research and clinical studies across all disease areas. The manuscript management system is completely online and includes a very quick and fair peer-review system, which is all easy to use. Visit http://www.dovepress.com/ testimonials.php to read real quotes from published authors. 